\title{
Candida albicans isolated from post-operative penetrating keratoplasty patient: Case report
}

\author{
Abhishek Chandra', Munesh Kumar Gupta², Ragini Tilak ${ }^{2}$ \\ Department of Ophthalmology, Institute of Medical Sciences, Banaras Hindu University, Varanasi, India, Department of Microbiology, Institute \\ of Medical Sciences, Banaras Hindu University, Varanasi, India
}

\section{A B S T R A C T}

We report a case report of Candida albicans suture infiltrate on 3rd post-op day in a 53 year female operated for penetrating keratoplasty. Candida albicans was identified by $\mathrm{KOH}$ mount, Gram Staining, germ tube, growth at 450C, chlamydospore formation and light green color on CHROMagar with sugar assimilation and culture characteristics. Despite being susceptible to Fluconazole by broth microdilution, patient did not respond to $0.3 \%$ fluconazole eye drops. On antifungal susceptibility testing by CLSI44A, it was susceptible to only Amphotericin B (100units). Patient was then started on $0.15 \%$ fortified amphotericin B eye drops resulting in complete resolution of infiltrates.

Key words: Post-keratoplasty, Candida, Fluconazole, Amphotericin B, Penetrating keratoplasty

\section{INTRODUCTION}

Post-keratoplasty infections are the most frequent complications associated with graft failure. ${ }^{1}$ Most of these infections are caused by bacteria with rarely caused by fungi. Prompt identification and treatment is needed to prevent the graft failure. We are presenting a case of post-keratoplasty suture infiltrate, caused by Candida albicans that showed no response to $0.3 \%$ fluconazole eye drop. This case report shows the importance of proper microbiological evaluation for good clinical outcome.

\section{CASE PRESENTATION}

A 53 year old female, from a rural area of Northern India, presented in Ophthalmology OPD with complain of diminished vision in left eye for more than ten years. She gave no history of trauma or pain in her eyes. Her visual acuity was limited to finger counting close to face. On examination, she had corneal opacity in her left eye. Anterior chamber (AC) was deep, there was no inflammation in her eye, the lens was clear but fundus was not visible. Intraocular pressure and ocular movements were normal.
The patient was advised to undergo keratoplasty and she underwent penetrating keratoplasty which was uneventful (Figure 1a). The donor rim was sent for microbiological investigation which was negative for any microorganism. Patient was discharged on topical antibiotic and corticosteroid eye drops.

On $3^{\text {rd }}$ post-operative day, the patient complained of gritty sensation in affected eye. On slit lamp examination, there was early suture infiltrate at 5 o'clock position (Figure 1b). Infiltrated suture was removed. Corneal infiltrates were scraped and microbiological work up including Gram stain, $\mathrm{KOH}$ wet mount and inoculation on Blood agar and Sabouraud's Dextrose agar was carried out. Gram stain revealed budding yeast cells and pus cells. (Figure 2a) $\mathrm{KOH}$ wet mount showed budding yeast cells with pseudohyphae. (Figure 2b) Topical fluconazole $0.3 \%$ eye drop on hourly basis was prescribed but despite continuous instillation of antifungal eye drops, the corneal infiltrates increased in size and depth (Figure 1c).

On $6^{\text {th }}$ post-operative day the anti-fungal susceptibility showed that the isolated Candida albicans was resistant to fluconazole, itraconazole and voriconazole, being sensitive to only amphotericin B. On the basis of this antifungal 
susceptibility, topical fortified amphotericin B $0.15 \%$ eye drop were administered on hourly basis. Corneal infiltrates decreased in size and intensity and patient showed improvement. After a week of amphotericin instillation there was minimal infiltrates present at 5 O'clock position (Figure 1d) and at two weeks there was complete resolution of infiltrates.

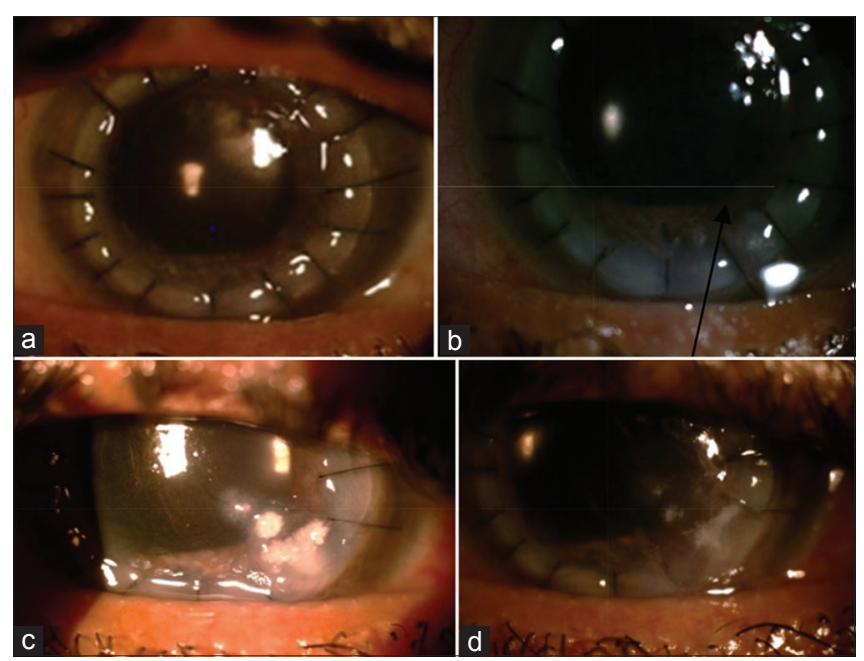

Figure 1: Showing sequential photographs of cornea after keratoplasty (a) $1^{\text {st }}$ post operative day-no suture infiltrate (b) Suture infiltrates $3^{\text {rd }}$ post operative day (c) Suture infiltrates after $0.3 \%$ fluconazole eye drop (d) Suture infiltrates after $0.15 \%$ amphotericin B eye drop
After overnight incubation on Sabouraud's Dextrose agar, colonies were small with irregular margins along the streaking in "C" shape. (Figure 2c) Gram stain was performed from the culture growth, revealed gram positive budding yeast cells. (Figure 2d) Cultured cells were incubated in human serum at $37^{\circ} \mathrm{C}$ and examined under low power where it showed the germ tube (Reynolds- Braude Phenomenon) (Figure 2e). ${ }^{2}$ ${ }^{4}$ Isolated organism also grew at $45^{\circ} \mathrm{C}$. (Figure $2 \mathrm{f}{ }^{5}$ Isolated organism fermented glucose, sucrose and Maltose, but not lactose by changing color to Red. On cornmeal agar, isolated yeast produced thick, terminal chlamydospores. (Figure 2g) ${ }^{6}$ On CHROMagar, after overnight incubation at $37^{\circ} \mathrm{C}$ there is light green colour. (Figure 2h). ${ }^{7}$ Ability to form Germ Tube, growth at $45^{\circ} \mathrm{C}$, chlamydospore production and light green color on CHROMagar confirmed that isolated organism was Candida albicans.

Antifungal susceptibility testing was performed against fluconazole (25 ug), itraconazole (10 ug), voriconazole (1 ug), and amphotericin B (100 units) by disc diffusion method. ${ }^{8}$ Minimum inhibitory concentration of these antifungal agents was determined against isolated yeast by broth microdilution method. ${ }^{9}$ The isolated organism was susceptible to only amphotericin B by disc diffusion method. (Figure 2i) Broth microdilution method showed

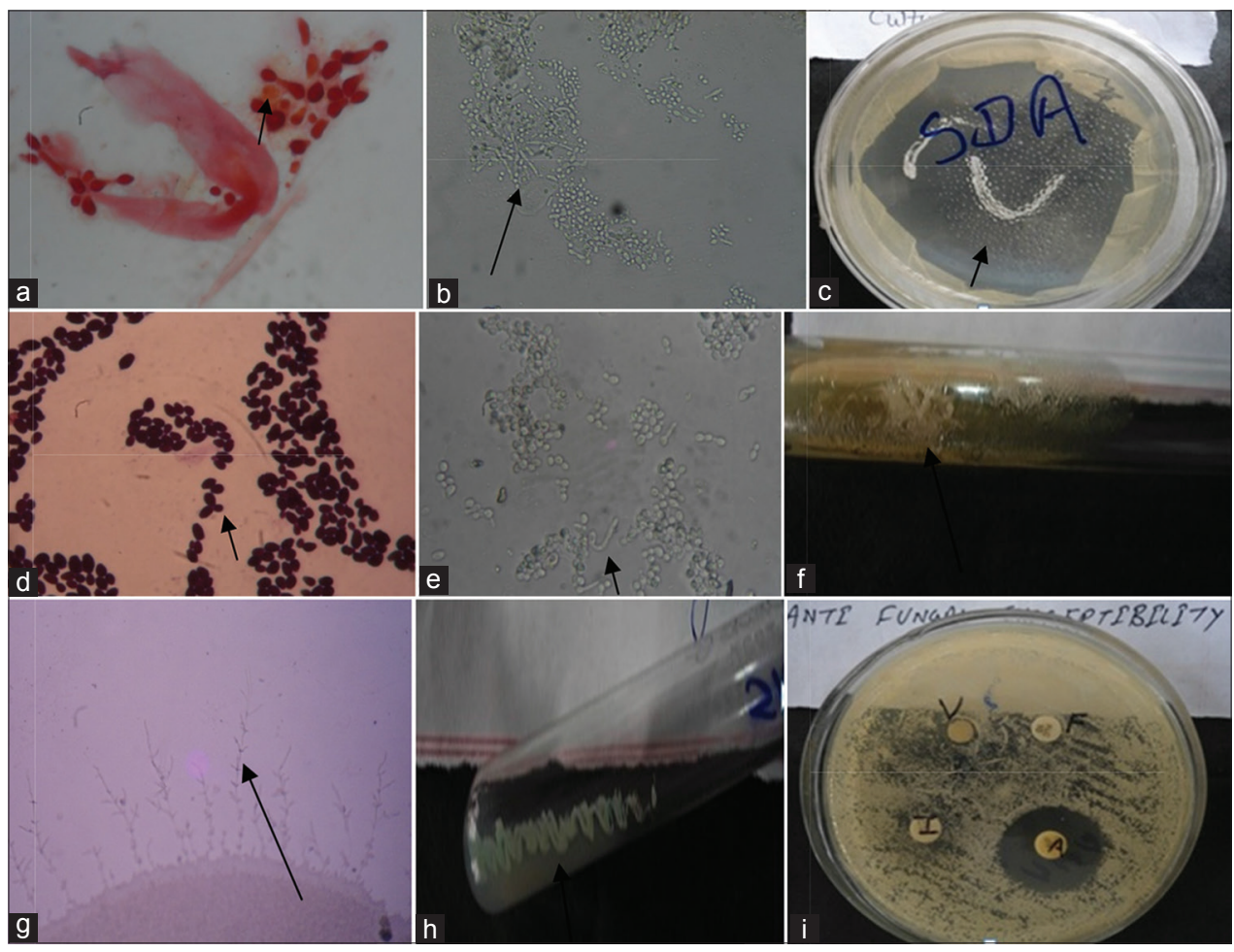

Figure 2: Microbiological test with identification and disc diffusion susceptibility testing (a) Gram's stain-Budding yeast cells (Corneal scrape) (b) $\mathrm{KOH}$ - budding yeast cells with pseudohyphae(scrape) (c) Growth on Sabouraud's Dextrose Agar (d) Gram's stain-Budding yeast cells (culture) (e) Germ tube production (f) Growth at $45^{\circ} \mathrm{C}(\mathrm{g}$ ) Thick terminal chlamydospores on Cornmeal agar (h) Light green colour Growth on CHROMagar (i) Antifungal susceptibility testing on Mueller Hinton Agar 
minimum inhibitory concentration (MIC) of 2, 0.5, 0.25 and $0.125 \mu \mathrm{g} / \mathrm{ml}$ for fluconazole, itraconazole, voriconazole and amphotericin B respectively.

\section{DISCUSSION}

Candida albicans, is commensal yeast, usually infect immuno-compromised patients especially those on prolonged antibiotic and corticosteroid drops. A substantial proportion of patients are colonized with Candida species after surgery, but only some of them subsequently develop invasive candidiasis. However, clinical signs of severe infection manifest only late, presenting a challenge for diagnosis. ${ }^{10}$

Candida is an infrequent microbe isolated from corneal ulcers in India. Upadhyay et al., from south India revealed Candida keratitis in $0.7 \%$ of fungal corneal ulcer in a series of 324 corneal ulcer patients. ${ }^{11}$ Bandyopadhyay $\mathrm{S}$ et al., reported Candida keratitis in 3\% cases with fungal corneal ulcer (12/399). ${ }^{12}$ On the other hand, a case series of 24 patients from Philadelphia Pennsylvania reported Candida albicans as most common fungal pathogen of corneal ulcer patients. ${ }^{13}$

Candida is rare cause of post-keratoplasty suture infiltrate. Topical instillation of antibiotics along with steroid favors Candida invasion. Keyhani $\mathrm{K}$ et al., has reported the development of post penetrating keratoplasty fungal infection in 4 patients out of 28 recipient patients. Candida was isolated from these all patients. ${ }^{14} \mathrm{MC}$ Caldwell et al., has reported a case of post penetrating keratoplasty Candida tropicalis endophthalmitis in 57 year old male. ${ }^{15}$ Post deep anterior lamellar keratoplasty (DALK) and descemet stripping and automated endothelial keratoplasty (DSAEK), Candida keratitis have been reported by MR Sedaghat ${ }^{16}$ and Koenig SB. ${ }^{17}$

Candida albicans is usually highly susceptible to fluconazole. A. Panda et al., reported that topical fluconazole is safe and effective antifungal agent in Candida keratitis. ${ }^{18}$ However, in our case, the isolated organism showed no response to $0.3 \%$ fluconazole eye drops as the isolated Candida albicans showed higher MIC against fluconazole $(2 \mu \mathrm{g} / \mathrm{ml})$. Increasing MIC of fluconazole against Candida albicans poses a greater challenge to treat these fungal infections.

\section{CONCLUSION}

Candidal infection in post-keratoplasty patient is rare. This case report shows the emergence of higher fluconazole MIC Candida albicans in post-keratoplasty patients. It is thus important for an Ophthalmologist to scrape all post-operative patients with corneal infiltrate and subject them to complete microbiological assessment including sensitivity to available topical drugs. This would not only lead to proper identification of micro-organism but would also provide us antibiotic susceptibility pattern. A more scientific approach would therefore lead to good treatment outcome and prevent consequences like endophthalmitis and graft failure.

\section{REFERENCES}

1. Confino $\mathrm{J}$ and Brown SI. Bacterial endophthalmitis associated with exposed monofilament sutures following corneal transplantation. Am J Ophthalmol 1985; 99:111-113.

2. Henry A. Bartegs, Gerson Cohen, and Irwin W. Scopp J DENT RES, March 1969; vol. 48, 2: pp. 230-235.

3. Reynolds R and Braude Al. The filament inducing property of blood for Candida albicans: Its nature and significance. Clin Res Proc 1956; 7: 417-420.

4. Braude Al, Davis CE and Fierer J. Infectious diseases and medical microbiology. 2nd ed. W.B. Saunders. Philadelphia, 1986; pp. 571-579.

5. Pinjon E, Sullivan D, Salkin I, Shanley D and Coleman D. Simple, inexpensive, reliable method for differentiation of Candida dubliniensis from Candida albicans. J Clin Microbiol 1998;36(7):2093-2095.

6. Gow, NAR. Cell biology and cell cycle of Candida, 2002; p. 145158.

7. Beighton D, Ludford R, Clark DT, Brailsford SR, Pankhurst CL, Tinsley GF, et al. Use of CHROMagar Candida medium for isolation of yeasts from dental samples J Clin Microbiol 1995, 33:3025-302.

8. NCCLS (2004) Reference method for antifungal disk diffusion susceptibility testing of yeasts; approved guideline. NCCLS document M44-A. National Committee for Clinical Laboratory Standard, Wayne.

9. CLSI (2008a) Reference method for broth dilution antifungal susceptibility testing of yeasts; approved standard-third ed; CLSI document M27-A3. Clinical and Laboratory Standards Institute, Wayne.

10. Eggimann P. PITTETD, post operative fungal infection surg infect 2006;7.

11. Upadhyay MP, Karmacharya PC, Koirala S, Tuladhar NR, Bryan LE, Smolin G, et al. Epidemiologic characteristics, predisposing factors, and etiologic diagnosis of corneal ulceration in Nepal. Am J Ophthalmol.1991 Jan 15;111(1):92-9.

12. Bandyopadhyay S, Das D, Mondal KK, Ghanta AK, Purkrit SK and Bhasrar R. Epidemiology and laboratory diagnosis of fungal corneal ulcer ion Sundarban region of West Bengal, Eastern India. Nepal J Ophthalmol 2012;4(7):29-36.

13. Tanure MA, Cohen EJ, Sudesh S, Rapuano CJ and Laibson PR. spectrum of fungal keratitis at Wills EYE Hospital Philadelphia, Pennylvania. Cornea 2000;19:307-312.

14. Keyhani K, Seedor JA, Shah MK, Terraciano AJ and Ritterband DC. The incidence of fungal keratitis and endophthalmitis following penetrating keratoplasty. Cornea. 2005 Apr;24(3):288-291.

15. Caldwell MC, Perfect JR, Carlson AN and Proia AD. Candida glabrata endophthalmitis following penetrating keratoplasty. J Cataract Refract Surg. 2009 Mar;35(3):598-602. 
16. Mohammad R Sedaghat and Hosseinpoor SS. Candida albicans interface infection after deep anterior lamellar keratoplasty. Indian J Ophthalmol 2012;60(4):328-330.

17. Koenig SB, Wirostko WJ, Fish RI and Vovert DJ. Candida keratitis after descemet stripping and automated endothelial keratoplasty. Cornea 2009; 28(4):471-473.

18. Panda A, Sharma $\mathrm{N}$ and Angra SK. Topical fluconazole therapy of Candida keratitis. Cornea 1996;15(4):373-375. Authors Contribution:
AC: Concept and design of the study, definition of intellectual content, literature search, clinical studies, data acquisition, manuscript preparation, editing and
review; MKG: Concept and design of the study, literature search, clinical studies, data acquisition, manuscript preparation; RT: Definition of intellectual content, literature search, manuscript editing and review.

Source of Support: Nil, Conflict of Interest: None declared. 